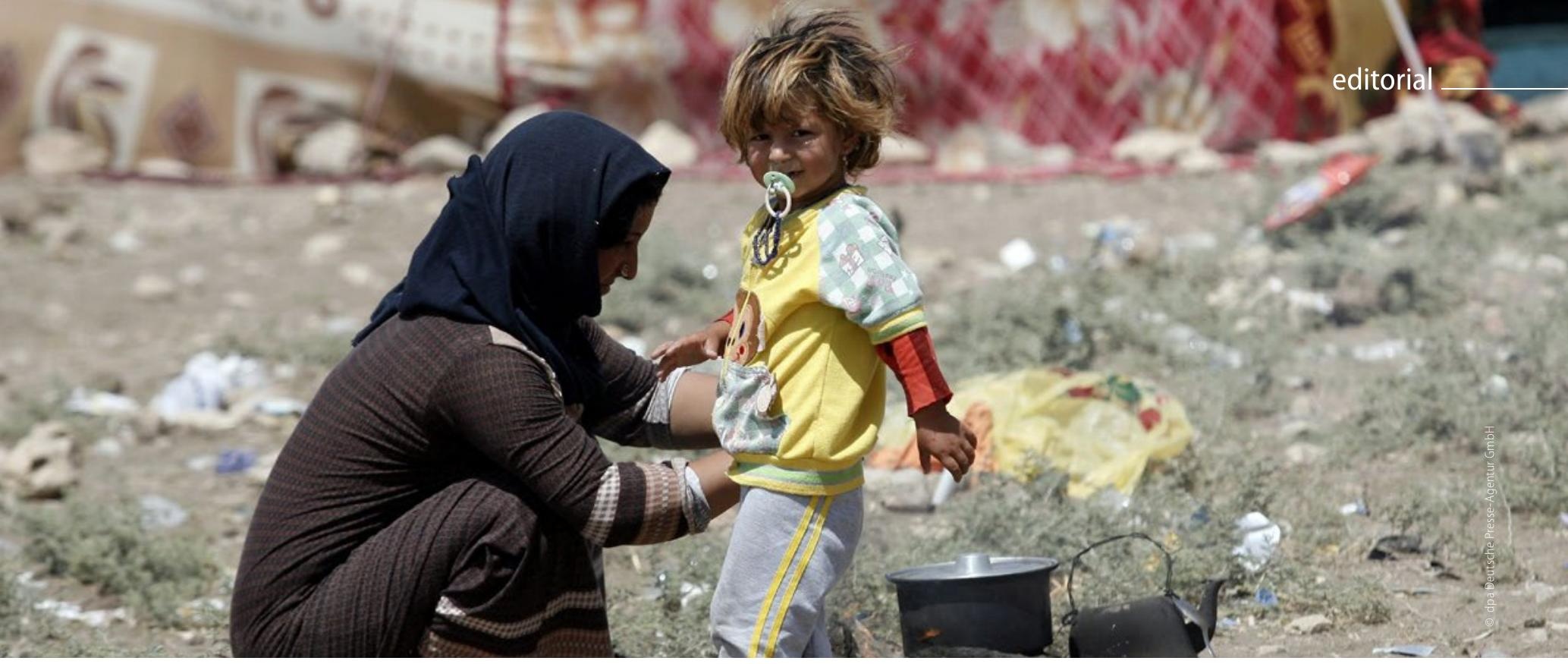

\title{
Prävention und Therapie von psychiatrischen Traumafolgeerkrankungen bei Flüchtlingen
}

Bereits in diesem Jahr werden in Deutschland annähernd eine Million Flüchtlinge und Asylbewerber - hauptsächlich aus Kriegsgebieten - erwartet. Ein Großteil der Erwachsenen und Kinder hat kriegsbedingten Extremstress, Todesangst, Gewalt, Vergewaltigungen, Hass und Demütigungen erlebt. Allgemein geht man davon aus, dass solche Traumatisierungen bei bis zu $50 \%$ der Betroffenen eine psychiatrischen Folgeerkrankung wie posttraumatische Belastungsstörung (PTBS), Depression, Angst- oder Suchterkrankung nach sich ziehen können. Unbehandelt besteht eine hohe Gefahr zur Chronifizierung. Demnach muss man - vorsichtig geschätzt - mit 100.000 bis 200.000 Menschen mit einem entsprechenden Behandlungs- beziehungsweise Präventionsbedarf rechnen. Zwar gibt es sowohl für die Prävention als auch Behandlung von posttraumatischen Störungsbildern gut etablierte und evidenzbasierte psychotherapeutische Verfahren. Diese kommen jedoch wegen der Sprachund Kulturbarrieren und des in der Regel ungewöhnlich hohen zeitlichen Aufwands für Flüchtlinge nicht eins zu eins infrage. Bei den jetzt von der Bundesregierung für 2016 eingeplanten Sondermitteln von drei Milliarden Euro sind Kosten für die Behandlung psychischer Folgen nach Extrembelastungen nicht erwähnt. Die Aufnahmerichtlinien für Asylbewerber schließen inzwischen zwar das Recht auf psychotherapeutische Behandlung ein, jedoch sind notwendige Anstrengungen, die erforderlichen Schritte einzuleiten, bisher nicht erkennbar. Wir brauchen auf jeden Fall eine große Zahl sogenannter geschulter Sprach- und Kulturmittler, die nicht durchweg diplomierte Dolmetscher sein müssen, sowie Therapeuten, die geeignete Präventions- und Therapieverfahren für traumatisierte Flüchtlinge und Asylbewerber durchführen können. Dabei dürften SteppedCare-Modelle und vor allen Dingen Kurzzeitverfahren unabdingbar sein.

Bisher gibt es nur wenige entsprechende und wissenschaftlich zumindest in Ansätzen untersuchte Verfahren. So hat Edna Foa

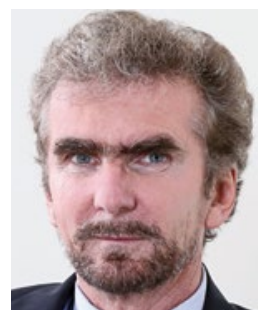

Prof. Dr. med. Mathias Berger

Ärztlicher Direktor der Klinik

für Psychiatrie und Psychotherapie

Universitätsklinikum Freiburg

E-Mail: mathias.berger@uniklinik-freiburg.de

für die Tsunami-Opfer eine Kurzform des „Prolonged Exposure" entwickelt, die auch von hierin ausgebildeten Nicht-Ärzten/ Psychologen anwendbar ist. Im Balkan-Krieg wurde mit dem Sarajewo-Protokoll eine EMDR-Variante entwickelt, die weitgehend auf sprachliche Kommunikation verzichtet. Für traumatisierte Menschen vor allen Dingen in Sri Lanka und Afrika wurden die Narrative Expositionstherapie (NET) sowie in Chile die Testimony-Therapie entwickelt und in ersten Studien evaluiert. Mehrere Gruppen erproben Internetprogramme zur Prävention und Therapie von Traumafolgestörungen in unterschiedlichen Sprach- und Kulturregionen.

Bei der hohen Relevanz der Thematik sollten wissenschaftliche Anstrengungen unternommen werden, die geeignetsten und praktikabelsten Präventions- und Therapieformen rasch zu evaluieren, weiterzuentwickeln und adäquate Formen der Implementierung zu ermitteln. All das wird nur gelingen, wenn die Bundesregierung die Problematik erkennt und Lösungen im notwendigen Rahmen finanziell fördert. Ansonsten ist damit zu rechnen, dass viele Flüchtlinge, die jetzt in Deutschland eine neue Heimat suchen, langfristig unter kriegsbedingten psychischen Folgen leiden und dies große Probleme bei der Eingliederung in einen neuen Lebensraum bedingen wird. Mathias Berger 